\title{
Quantitative Trait Loci Affecting Flag Leaf Development in Rice (Oryza sativa L.)
}

\author{
Sohei Kobayashi ${ }^{1,2)}$, Yoshimichi Fukuta*2), Satoshi Morita ${ }^{3)}$, Tadashi Sato ${ }^{4}$, Mitsuru Osaki ${ }^{1)}$ \\ and Gurdev S. Khush' \\ 1) Graduate School of Agriculture, Hokkaido University, Kita-9 Nishi-9, Kita, Sapporo, Hokkaido 060-8589, Japan \\ 2) International Rice Research Institute, DAPO Box 7777, Metro Manila, Philippines \\ 3) Agriculture, Forestry and Fisheries Research Council, The Ministry of Agriculture, Forestry and Fisheries of Japan, 1-2-1 Kasumi- \\ gaseki, Chiyoda, Tokyo 100-8950, Japan \\ 4) Graduate School of Life Science, Tohoku University, Katahira, Aoba, Sendai, Miyagi 980-8577, Japan
}

One hundred and ninety-one recombinant inbred lines (RILs) $\left(\mathrm{F}_{7}\right)$ derived from a cross between Milyang 23 (M23) and Akihikari (AK) were grown in 1997 in Joetsu, Japan (temperate zone), and during the 2000-01 dry and wet seasons (four consecutive seasons) in Los Baños, Philippines (tropical zone) to detect quantitative trait loci (QTLs) for flag leaf length, width and angle (FLL, FLW and FLA) of rice (Oryza sativa L.). The detected QTLs suggested that flag leaf development was influenced by nine genomic regions categorized into three groups. In Group I, three regions (chromosomes 1, 4 and 6) increased both FLL and FLW. The QTL profiles showed that the effects of these three regions were powerful and stable across locations. Especially, the effect of the region on chromosome 4 (the nearest RFLP marker, $X N p b 235$ ) accounted for 9-17\% and 20-26\% of the FLL and FLW variances, respectively. In Group II, four regions (chromosomes 2, 3, 10 and 11) affected FLL, whereas in Group III, two regions (chromosomes 8 and 12) affected FLW. The detection pattern of QTLs showed that three regions in Groups II and III (chromosomes 3, 10 and 12) were expressed by the growth conditions of Los Baños. No region with a clear effect on FLA was identified, although this trait was segregated largely in the RILs. By characterizing the nine regions in detail, this paper elaborates on the genetic factors and mechanisms controlling flag leaf development.

Key Words: Oryza sativa L., rice, flag leaf, quantitative trait locus (QTL), recombinant inbred line (RIL).

\section{Introduction}

In cereal crops, the flag leaf produces a large proportion of the carbohydrates stored in grains (Gladun and Karpov 1993, Li et al. 1998). In rice (Oryza sativa L.), the improvement of flag leaf traits through plant breeding has led to a

Communicated by K. Okuno

Received March 3, 2003. Accepted May 14, 2003.

*Corresponding author (e-mail: y.fukuta@cgiar.org) drastic increase in grain yield (Yonezawa 1997). Flag leaf traits, such as flag leaf length, width and angle (FLL, FLW and FLA, respectively), are inherited quantitatively and influenced largely by growth environments (Yamagata 1997). Because of the trait characteristics, it had been difficult to identify the genetic factors (loci) responsible for each trait and to analyze the genetic mechanisms of flag leaf development in rice.

The recent development of molecular markers has enabled to identify gene loci affecting quantitative traits, such as days to heading, culm length (CL) and panicle number $(\mathrm{PnN})$, on a linkage map in rice (Yano and Sasaki 1997). The response of such an agronomically important quantitative trait locus (QTL) to the growth environments has been demonstrated by Yan et al. (1999), Yamamoto et al. (2000) and Kobayashi et al. (2003) who conducted QTL analyses across different locations. However, compared with those major quantitative traits, flag leaf traits had seldom been examined despite their importance. Li et al. (1999) examined FLA in the Lemont/Teqing $\mathrm{F}_{2}$ population and located five QTLs on chromosomes 2, 5, 6, 7 and 9. Yan et al. (1999) grew the IR64/Azucena doubled haploid (DH) population under subtropical and temperate zones, and detected seven, seven and six QTLs for FLL, FLW and FLA, respectively. Results from the latter QTL analysis showed the presence of a genomic region affecting both FLL and FLW on chromosome 4 and regions affecting the flag leaf trait at only one location on chromosomes 2, 4, 6, 9, 11 and 12 . These regions, which have multiple effects or are expressed by a particular environment, possibly play important roles in the genetic control of flag leaf development (Li et al. 1995, Lin et al. 1996, Yan et al. 1999). Further studies should be carried out to locate and characterize such regions using different mapping populations.

Milyang 23 (M23) is an Indica-type variety, whereas Akihikari (AK) is a Japonica-type variety. M23 has a long, wide and erect flag leaf, compared with AK. Since these two varieties have been used in actual breeding programs (Yokoo et al. 1994), QTL mapping using the M23/AK cross would possibly disclose a typical genetic nature (background) underlying the different types of flag leaf development (Yano and Sasaki 1997). In this study, recombinant inbred lines (RILs) derived from the M23/AK cross (Fukuta et al. 1999) were grown during the cropping season in Joetsu, 
Japan (temperate zone), and during the dry and wet seasons in Los Baños, Philippines (tropical zone), to map QTLs for FLL, FLW and FLA. Through the QTL mappings, chromosomal regions with multiple effects on the flag leaf traits or responsive to the environments were studied to analyze the genetic mechanism of flag leaf development in rice.

\section{Materials and Methods}

\section{Plant materials and collection of phenotypic data}

One hundred and ninety-one RILs ( $\mathrm{F}_{7}$ generation) derived from the M23/AK cross (Fukuta et al. 1999) were grown under irrigated field conditions from May to October in 1997 (abbreviated as 97J) at the former Hokuriku National Agricultural Experiment Station (HNAES, currently Hokuriku Research Center, Long. $138^{\circ}$ E, Lat. $38^{\circ} \mathrm{N}$ ), Joetsu, Japan, and during the dry (Jan-Apr) and wet (JunSep) seasons in 2000 and 2001 (abbreviated as 00DS, 00WS, $01 \mathrm{DS}$ and $01 \mathrm{WS}$ ) at the International Rice Research Institute (IRRI, Long. $121^{\circ} \mathrm{E}$, Lat. $14^{\circ} \mathrm{N}$ ), Los Baños, Philippines. Joetsu and Los Baños are located in temperate and tropical zones, respectively, with an annual rainfall $>2000 \mathrm{~mm}$. Plants were arranged as a single planting per hill at the locally accepted densities: 15.4 plants $\mathrm{m}^{-2}$ in Joetsu and 16.7 plants $\mathrm{m}^{-2}$ in Los Baños. For the control of pests, insects and water, and for fertilizer application, conventional field management was adopted, based on the HNAES and IRRI experimental farm practices. More than five plants were harvested 35 days after heading (when the plants were matured) to measure the length, width (widest part) and angle (from the panicle side) of three flag leaves per plant. FLL was measured in all the seasons, FLW in 97J, 00WS, 01DS and $01 \mathrm{WS}$, and FLA in 97J and 00DS.

\section{QTL mapping}

Using 182 restriction fragment length polymorphism (RFLP) markers, Fukuta et al. (1999) determined the genotypes of marker loci and constructed a complete linkage map (Fig. 2). With the genotypic data, interval mapping was performed using the computer software $Q G E N E$ ver. 3.05 (Nelson 1997) to identify QTLs through the following two steps. For the first step, all the visible LOD peaks $>2.0$ were located, including that near the highest peak. This threshold is suitable for detecting relatively minor-effect genes in avoiding a false positive effect (Yano and Sasaki 1997). For the second step, the LOD peaks $(>2.0)$, of which the nearest RFLP marker showed $P<0.01$, were accepted as QTLs. This threshold has also been commonly used in recent singlepoint QTL analyses (Yano and Sasaki 1997). Moreover, we only focused on the genomic regions that repeatedly showed QTLs for a trait across crop seasons to confirm our analysis.

\section{Results}

\section{Trait segregation}

For FLL and FLW, the values of the M23 plants (26.3-
31.2 and $1.48-1.70 \mathrm{~cm}$, respectively) were consistently higher than those of the AK plants (18.6-27.9 and $1.06-1.43 \mathrm{~cm})$, whereas for FLA, the values of the M23 plants (0-6.0 degree) were lower than those of the AK plants (27.5-36.7 degrees) (Fig. 1). These results confirmed that the M23 plants have a long, wide and erect flag leaf compared with the AK plants. For all the three traits, wide or continuous frequency distributions with transgressive segregants were observed. The FLL of 00DS, 01DS and 01WS showed a binominal or distorted distribution, indicating the involvement of a relatively powerful gene(s). Similar distributions were also observed for FLW in 00WS and 01WS, and FLA in 97J.

The mean values of FLL tended to be higher in the Los Baños wet seasons (00WS and 01WS) $(26.7-27.0 \mathrm{~cm})$ than in the other three seasons (97J, 00DS and 01DS) (24.1-25.9 $\mathrm{cm})$. On the other hand, the mean values of FLW were clearly higher in Joetsu $(97 \mathrm{~J})(1.49 \mathrm{~cm})$ than in Los Baños $(00 \mathrm{WS}$, $01 \mathrm{DS}$ and $01 \mathrm{WS})(1.24-1.31 \mathrm{~cm})$. For FLA, the mean value was higher in Los Baños (00DS) (24.5 degrees) than in Joetsu (97J) (14.9 degrees). These results indicate that each trait differed depending on the growth environments, especially between tropical and temperate climates.

\section{Trait correlation}

FLL was positively correlated between seasons (Table 1). The correlation coefficient ranged from 0.54 to 0.73 among the Los Baños seasons and from 0.39 to 0.51 across the Joetsu and Los Baños seasons. Accordingly, FLL showed a high repeatability in values across seasons, particularly in Los Baños. Similar correlations and trends were also observed for FLW ( $R=0.78-0.81$ in Los Baños and 0.69-0.77 across the two locations). The coefficients of FLW were clearly higher than those of FLL. In the case of FLA, the correlation coefficient of 97J with 00DS was 0.35 .

FLL and FLW were positively correlated in a season or across seasons. The coefficients tended to be more significant in Los Baños (0.33-0.65) and in Joetsu (0.48) than across the two locations (0.28-0.37). Accordingly, these two flag leaf traits were genetically related, especially at a location. However, such a genetic relation was not clearly observed between FLA and FLL or FLW ( $R=-0.09$ to 0.16$)$.

\section{QTLs for FLL, FLW and FLA}

Throughout the five cropping seasons (four for FLW and two for FLA), a total of 32, 24 and six QTLs were detected for FLL, FLW and FLA, respectively (Fig. 2 and Table 2). For FLL, FLW and FLA, the phenotypic variance explained (PVE) by each QTL was 5.1-17.3\%, 5.3-26.0\% and $5.1-9.5 \%$, respectively. The total PVE by these QTLs was $29.5-45.7 \%, 44.5-47.6 \%$ and $11.6-20.0 \%$, and the additive effect of QTL was $0.8-2.1 \mathrm{~cm}, 0.04-0.09 \mathrm{~cm}$ and $1.54-2.97$ degrees. Most the QTLs were mapped onto the nine genomic regions designated as R1, R2, R3, R4, R6, R8, R10, R11 and R12 in Fig. 2.

For FLL, seven, four, six, seven and eight QTLs were detected for $97 \mathrm{~J}, 00 \mathrm{DS}, 00 \mathrm{WS}, 01 \mathrm{DS}$ and $01 \mathrm{WS}$, respectively 

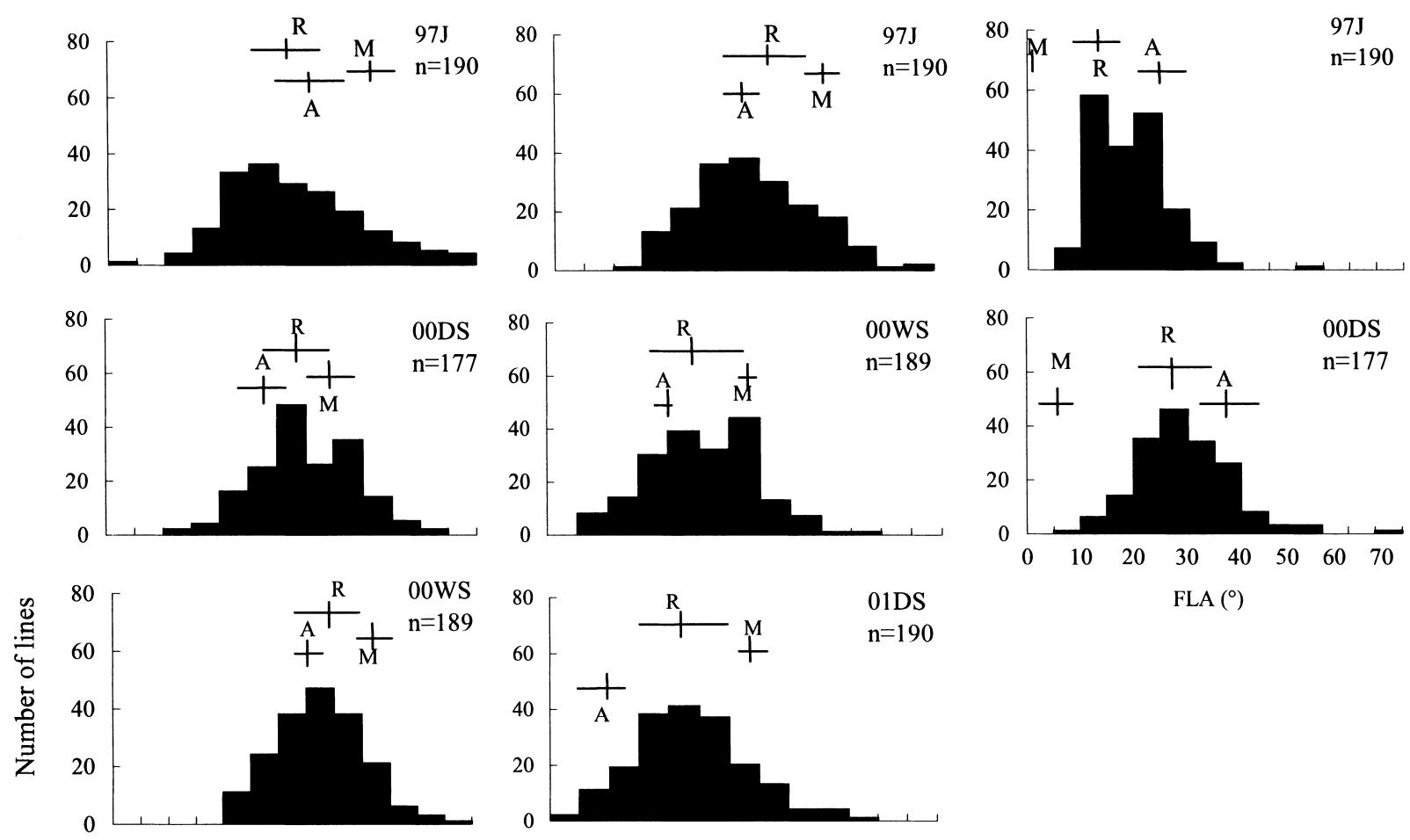

$01 \mathrm{DS}$
$\mathrm{n}=190$
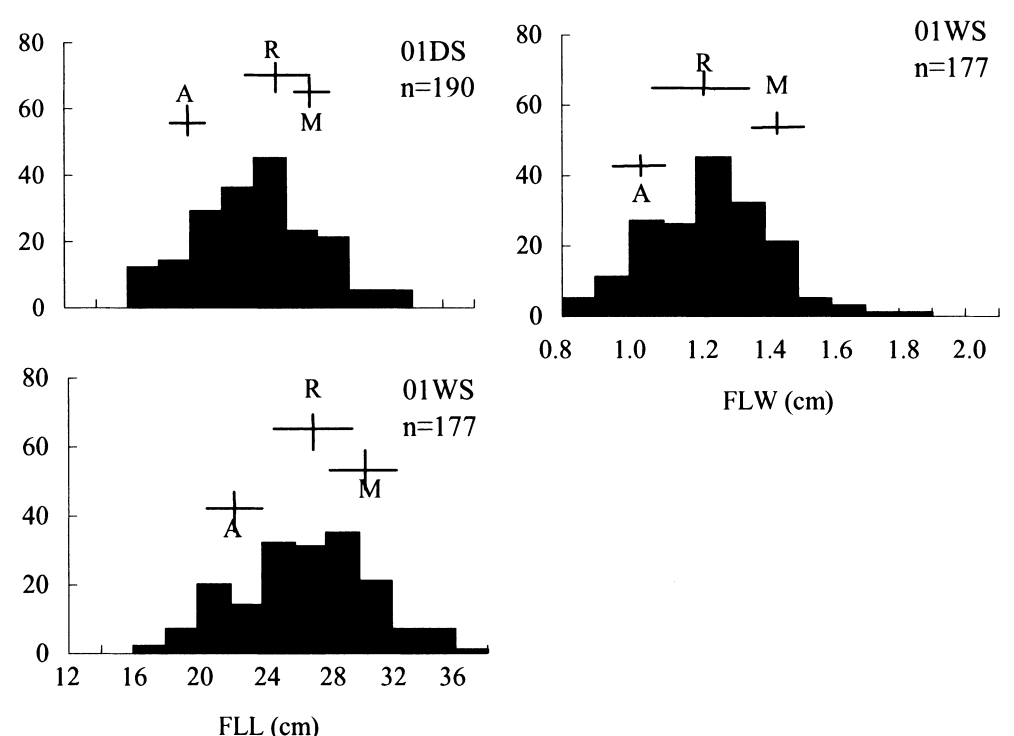

FLW (cm)

Fig. 1. Frequency distribution of flag leaf length, width and angle (FLL, FLW and FLA, respectively) in the Milyang 23/Akihikari RILs grown in 1997 (97J) in Joetsu, Japan, and during the 2000-2001 dry and wet seasons (00DS, 00WS, 01DS and 01WS) in Los Baños, Philippines. Mean value is shown with standard deviation (horizontal bar) for Milyang 23 (M), Akihikari (A) and RILs (R).

(Table 2). As shown in Fig. 2, QTLs were repeatedly found in seven regions: R1 (the nearest marker, XNpb359-C955) on chromosome 1, R2 (G1234) on chromosome 2, R3 (C136) on chromosome 3, R4 (XNpb235) on chromosome 4, R6 (XNpb135) on chromosome 6, R10 (C16) on chromosome 10 and R11 (C1172) on chromosome 11. In R1, QTLs were clearly found at two different positions near XNpb359 and C955. QTLs in R1, R2, R3, R6 and R10 increased FLL with the M23 allele, whereas those in R4 and R11 decreased it. In each season, the QTL in R4 tended to be most powerful
$(\mathrm{LOD}=3.5-7.1, \mathrm{PVE}=8.6-17.0 \%)$, followed by those in $\mathrm{R} 6$ $(\mathrm{LOD}=2.1-4.9, \mathrm{PVE}=5.2-11.2 \%)$ and in $\mathrm{R} 11(\mathrm{LOD}=2.0$ $3.9, \mathrm{PVE}=5.1-9.6 \%$ ). In R4, R6 and R11, QTLs were detected for all the five seasons, whereas in R3 and R10, QTLs were detected only for the Los Baños seasons.

For FLW, five, five, seven and seven QTLs were detected for 97J, 00WS, 01DS and 01WS, respectively. QTLs were repeatedly found in five regions: R1 (the nearest marker, XNpb359-C955) on chromosome 1, R4 (XNpb235) on chromosome 4, R6 (XNpb135) on chromosome 6, R8 
Table 1. Correlation coefficients among flag leaf length, width and angle (FLL, FLW and FLA, respectively) in the Milyang 23/Akihikari RILs grown in 1997 (97J) in Joetsu (JE), Japan, and during the 2000-2001 dry and wet seasons (00DS, 00WS, 01DS and 01WS) in Los Baños (LB), Philippines

\begin{tabular}{|c|c|c|c|c|c|c|c|c|c|c|c|c|c|}
\hline & \multirow{3}{*}{ Trait } & & \multicolumn{5}{|c|}{ FLL } & \multicolumn{4}{|c|}{ FLW } & \multicolumn{2}{|c|}{ FLA } \\
\hline & & & \multirow{2}{*}{$\begin{array}{l}\mathrm{JE} \\
97\end{array}$} & \multicolumn{4}{|c|}{ LB } & \multirow{2}{*}{$\begin{array}{l}\mathrm{JE} \\
97\end{array}$} & \multicolumn{3}{|c|}{ LB } & \multirow{2}{*}{$\begin{array}{l}\mathrm{JE} \\
97\end{array}$} & \multirow{2}{*}{$\begin{array}{c}\text { LB } \\
\text { 00DS }\end{array}$} \\
\hline & & & & 00DS & 00WS & 01DS & 01WS & & 00WS & 01DS & 01WS & & \\
\hline \multirow[t]{5}{*}{ FLL } & $\mathrm{JE}$ & 97 & 1.00 & & & & & & & & & & \\
\hline & LB & 00DS & 0.48 & 1.00 & & & & & & & & & \\
\hline & & 00WS & 0.43 & 0.63 & 1.00 & & & & & & & & \\
\hline & & 01DS & 0.51 & 0.65 & 0.55 & 1.00 & & & & & & & \\
\hline & & $01 \mathrm{WS}$ & 0.39 & 0.64 & 0.54 & 0.73 & 1.00 & & & & & & \\
\hline \multirow[t]{4}{*}{ FLW } & $\mathrm{JE}$ & 97 & 0.48 & 0.30 & 0.31 & 0.34 & 0.28 & 1.00 & & & & & \\
\hline & LB & $\overline{00 W S}$ & 0.36 & 0.42 & 0.48 & 0.37 & 0.33 & 0.77 & 1.00 & & & & \\
\hline & & 01DS & 0.35 & 0.48 & 0.41 & 0.65 & 0.48 & 0.72 & 0.78 & 1.00 & & & \\
\hline & & $01 \mathrm{WS}$ & 0.37 & 0.48 & 0.35 & 0.51 & 0.64 & 0.69 & 0.81 & 0.81 & 1.00 & & \\
\hline \multirow[t]{2}{*}{ FLA } & $\mathrm{JE}$ & 97 & $0.16^{* *}$ & $-0.03 *$ & $-0.09 *$ & $-0.03 *$ & $-0.05 *$ & $0.05^{*}$ & $-0.05^{*}$ & $-0.06^{*}$ & $-0.05^{*}$ & 1.00 & \\
\hline & LB & 00DS & $0.04 *$ & $0.08^{*}$ & $-0.07 *$ & $0.02 *$ & $0.06^{*}$ & $0.01 *$ & $0.03 *$ & $0.00^{*}$ & $0.09^{*}$ & 0.35 & 1.00 \\
\hline
\end{tabular}

*** and no symbol: no statistical significance, and $5 \%$ and $1 \%$ levels of significance, respectively.

(G2132B) on chromosome 8 and R12 (G1106) on chromosome 12. In R1, QTLs were found at two different positions, as in the case of FLL. QTLs in R1 and R6 increased FLW with the M23 allele, whereas those in R4, R8 and R12 decreased it. In each season, the QTL in R4 tended to be the most powerful (LOD $=9.1-12.4, \mathrm{PVE}=19.7-26.0 \%$ ), followed by those in R1 ( $\mathrm{LOD}=2.7-5.4, \mathrm{PVE}=6.7-12.3 \%)$ and in $\mathrm{R} 6(\mathrm{LOD}=2.3-3.6, \mathrm{PVE}=5.3-8.4 \%)$. In R1, R4 and R6, QTLs were detected for all the five seasons, whereas in R12, QTLs were detected only for the Los Baños seasons.

For FLA, two and four QTLs were detected for 97J and 00DS, respectively. No chromosomal region showed QTLs repeatedly across seasons. Most of the QTLs were not powerful and were located at different positions from those for FLL and FLW.

\section{Discussion}

In rice, the flag leaf plays an important role in supplying carbohydrates to grains (Gladun and Karpov 1993, Li et al. 1998). By using the M23/AK RILs, this study enabled to identify nine genomic regions affecting flag leaf development (R1, R2, R3, R4, R6, R8, R10, R11 and R12). Among these nine regions, R4 increased FLL and FLW (flag leaf size) with the AK allele for all the five seasons in Joetsu and Los Baños. The LOD-score curve showed that the QTL of these two traits was present near XNpb235 in R4. The multiple effect of R4 was powerful and stable across growth environments, compared with others. These results suggest that $\mathrm{R} 4$ exerts a considerable effect on the flag leaf size, which is not affected by the environments. The effect on FLW (av. $\mathrm{PVE}=23.3 \%$ ) was clearly larger than that on FLL (av. PVE $=11.7 \%$ ). The larger effect explains the higher total PVE and repeatability of FLW, compared with those of FLL (Table 1 and Table2). The repeatedly detected effects (QTL) also explain the high repeatability (positive correlation) in
FLL or FLW across seasons, and the positive genetic relation between FLL and FLW.

Using the IR64/Azucena DH population, Yan et al. (1999) located a QTL, which increased the flag leaf size (FLL and FLW) with the Azucena allele, between the RFLP markers $R G 163-R G 590$. This marker interval was located at $22.6-46.3 \mathrm{cM}$ from the long arm terminal of chromosome 4 based on the high-density linkage map of Causse et al. (1994). The QTL of R4 (XNpb235) was located at 15.7-28.1 $\mathrm{cM}$ from the long arm terminal of chromosome 4. These two QTLs identified by different studies were similar in their function, position and positive effect of the Japonica allele, suggesting that they are identical. These results indicate that the QTL of R4 is used in other rice varieties, and that its genotype is an important criterion for molecular marker selection to modify the flag leaf size. In R4, QTLs for PnN and panicle length (PnL) have been reported using the M23/AK RILs (Kobayashi et al. 2003). The QTLs for PnN and PnL were not as powerful as those for FLL and FLW, suggesting that R4 primarily affects the flag leaf size.

QTL for the flag leaf size was also identified in R1 (XNpb359-C955) and R6 (XNpb12). In R1, two different QTLs for the flag leaf size were located near $X N p b 359$ and C955. Kobayashi et al. (2003) also detected two different QTLs for PnN near XNpb359 and XNpb90, and a QTL for PnL near C955, using the M23/AK RILs. Integration of these results suggests that R1 has three QTLs as follows: one for PnN and the flag leaf size near $X N p b 359$, one for PnN near $X N p b 90$, and one for PnL and the flag leaf size near C955. It is interesting that the two QTLs near XNpb359 and C955 exert a common effect on the flag leaf, but different effects on other traits. Kobayashi et al. (2003) also detected a powerful QTL for CL, PnL and PnN near XNpb12 in R6. The traits affected by this region (FLL, FLW, CL, PnL and $\mathrm{PnN}$ ) suggest that the function of the QTL near $X N p b 12$ was also different from those of the two QTLs near $X N p b 359$ and 

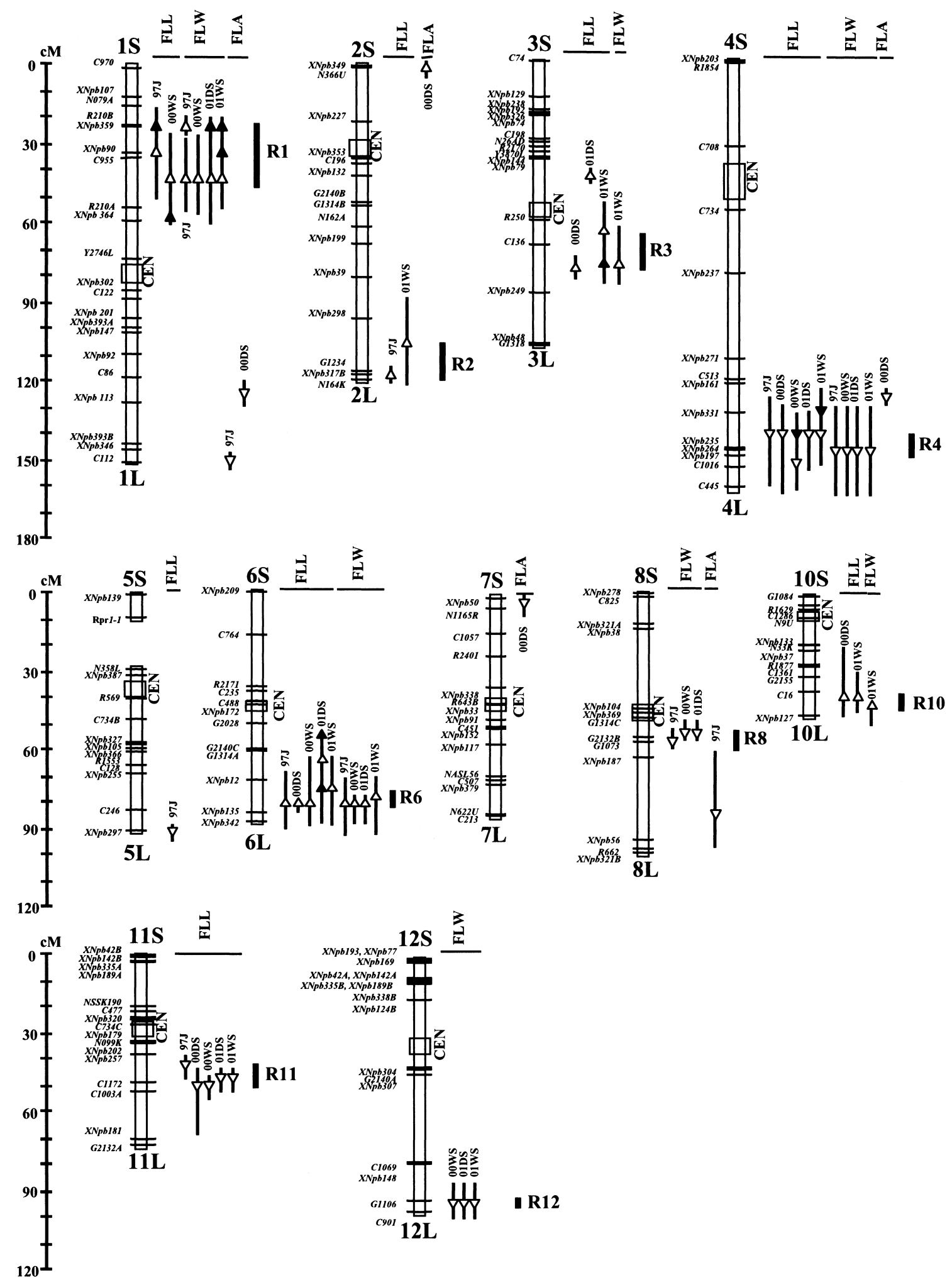

Fig. 2. Mapping of QTLs for flag leaf length, width and angle (FLL, FLW and FLA, respectively) in the Milyang 23/Akihikari RILs grown in 1997 (97J) in Joetsu, Japan, and during the 2000-2001 dry and wet seasons (00DS, 00WS, 01DS and 01WS) in Los Baños, Philippines. FLL was measured in all the seasons, FLW in 97J, 00WS, 01DS and 01WS, and FLA in 97J and 00DS. Vertical bars show the genomic regions recording LOD $>2.0$. Open and filled arrows denote the highest LOD peak and the neighboring one, respectively. Upward and downward arrows indicate that the Milyang 23 and Akihikari alleles increased the values, respectively. The genomic regions that repeatedly detected QTLs for a trait across seasons were shown by bold vertical bars and designated tentatively with the letter $\mathrm{R}$ on each chromosome number. 
Table 2. Location and effect of QTLs for flag leaf length, width and angle (FLL, FLW and FLA, respectively) in the Milyang 23/ Akihikari RILs grown in 1997 (97J) in Joetsu (JE), Japan and during the 2000-2001 dry and wet seasons (00DS, 00WS, 01DS and 01WS) in Los Baños (LB), Philippines

\begin{tabular}{|c|c|c|c|c|c|c|c|c|c|c|c|}
\hline \multirow{2}{*}{ Trait } & \multirow{2}{*}{ Location } & \multirow{2}{*}{ Season } & \multirow{2}{*}{ Chr. } & \multirow{2}{*}{$\begin{array}{l}\text { Position } \\
(\mathrm{cM})^{1)}\end{array}$} & \multirow{2}{*}{\multicolumn{2}{|c|}{ Marker interval2) $^{2}$}} & \multirow{2}{*}{$P^{3)}$} & \multirow{2}{*}{ LOD } & \multirow{2}{*}{$\begin{array}{c}\alpha \\
\left(\mathrm{cm} \mathrm{or}^{\circ}\right)^{4)}\end{array}$} & \multicolumn{2}{|c|}{$\mathrm{R}^{2}(\%)^{5)}$} \\
\hline & & & & & & & & & & Single & Multiple \\
\hline & \multirow{7}{*}{$\mathrm{JE}$} & \multirow{7}{*}{$97 \mathrm{~J}$} & 1 & 24 & N079A & $\underline{R 210 B}$ & $<0.001$ & 5.2 & 1.8 & 11.8 & \multirow{7}{*}{45.7} \\
\hline & & & 1 & 36 & $X N p b 359$ & $X N p b 90$ & $<0.001$ & 7.0 & 2.1 & 15.6 & \\
\hline & & & 2 & 120 & $G 1234$ & $\overline{X N p b 317 B}$ & $<0.001$ & 2.4 & 1.2 & 5.6 & \\
\hline & & & 4 & 148 & XNpb331 & XNpb235 & $<0.001$ & 6.2 & -2.0 & 14.0 & \\
\hline & & & $5 \mathrm{~L}$ & 67 & \multicolumn{2}{|c|}{ Xnpb297 } & $<0.001$ & 2.7 & -1.3 & 6.3 & \\
\hline & & & 6 & 80 & $X N p b 12$ & XNpb135 & $<0.001$ & 3.0 & 1.5 & 7.1 & \\
\hline & & & 11 & 43 & $X N p b 257$ & C1172 & $<0.001$ & 2.7 & -1.4 & 6.4 & \\
\hline & \multirow{25}{*}{ LB } & \multirow{4}{*}{ 00DS } & 3 & 75 & C136 & $X N p b 249$ & $<0.001$ & 2.7 & 1.1 & 6.7 & \multirow{4}{*}{30.2} \\
\hline & & & 4 & 149 & $X N p b 331$ & $\underline{X N p b 235}$ & $<0.001$ & 7.1 & -1.5 & 17.0 & \\
\hline & & & 6 & 81 & $X N p b 12$ & XNpb135 & 0.006 & 2.1 & 0.9 & 5.2 & \\
\hline & & & 11 & 51 & $\underline{C 1172}$ & $C 1003 A$ & $<0.001$ & 3.9 & -1.2 & 9.6 & \\
\hline & & \multirow{6}{*}{ 00WS } & 1 & 46 & C955 & $R 210 A$ & $<0.001$ & 4.0 & 1.2 & 9.4 & \multirow{6}{*}{29.5} \\
\hline & & & 1 & 61 & $R 210 A$ & XNpb364 & 0.002 & 2.5 & 0.8 & 5.8 & \\
\hline & & & 4 & 149 & $X N p b 331$ & XNpb235 & $<0.001$ & 3.7 & -1.0 & 8.7 & \\
\hline & & & 4 & 157 & C1016 & $C 445$ & $<0.001$ & 4.5 & -1.1 & 10.4 & \\
\hline \multirow{17}{*}{ FLL } & & & 6 & 81 & $\overline{X N p b 12}$ & $\underline{X N p b 135}$ & $<0.001$ & 4.2 & 1.1 & 9.7 & \\
\hline & & & 11 & 51 & $\underline{C 1172}$ & C1003A & $<0.001$ & 2.6 & -0.9 & 6.1 & \\
\hline & & \multirow{7}{*}{ 01DS } & 3 & 50 & $X N p b 144$ & $R 250$ & 0.007 & 2.1 & 1.1 & 4.9 & \multirow{7}{*}{37.1} \\
\hline & & & 4 & 148 & XNpb331 & XNpb235 & $<0.001$ & 3.7 & -1.2 & 8.6 & \\
\hline & & & 6 & 59 & $G 2028$ & $\overline{G 2140 C}$ & $<0.001$ & 4.0 & 1.2 & 9.2 & \\
\hline & & & 6 & 68 & $G 1314 A$ & $X N p b 12$ & $<0.001$ & 4.9 & 1.5 & 11.2 & \\
\hline & & & 6 & 76 & $\underline{X N p b 12}$ & $X N p b 135$ & $<0.001$ & 4.8 & 1.5 & 11.0 & \\
\hline & & & 10 & 45 & C16 & $X N p b 127$ & $<0.001$ & 7.8 & 1.8 & 17.3 & \\
\hline & & & 11 & 48 & $X N p b 257$ & $\underline{C 1172}$ & $<0.001$ & 2.4 & -1.0 & 5.7 & \\
\hline & & \multirow{8}{*}{$01 \mathrm{WS}$} & 2 & 106 & XNpb298 & G1234 & $<0.001$ & 3.5 & 1.5 & 8.6 & \multirow{8}{*}{39.4} \\
\hline & & & 3 & 67 & $R 250$ & C136 & 0.003 & 2.6 & 1.2 & 6.5 & \\
\hline & & & 3 & 78 & $\underline{C 136}$ & $X N p b 249$ & 0.003 & 2.5 & 1.3 & 6.3 & \\
\hline & & & 4 & 132 & $X N p b 161$ & XNpb331 & 0.004 & 2.4 & -1.2 & 6.1 & \\
\hline & & & 4 & 146 & $X N p b 331$ & XNpb235 & $<0.001$ & 3.5 & -1.4 & 8.7 & \\
\hline & & & 6 & 75 & $\underline{X N p b 12}$ & $X N p b 135$ & $<0.001$ & 4.5 & 1.6 & 11.0 & \\
\hline & & & 10 & 45 & C16 & $X N p b 127$ & $<0.001$ & 4.5 & 1.5 & 11.0 & \\
\hline & & & 11 & 48 & $X N p b 257$ & C1172 & 0.005 & 2.0 & -1.1 & 5.1 & \\
\hline
\end{tabular}

1) Distance from the terminal of the short arm.

2) The nearest RFLP marker to the QTL is underlined.

3) Probability of linkage between the QTL and the nearest marker.

4) Additive effect of the Milyang 23 allele compared with the Akihikari one.

5) $\mathrm{R}^{2}$ single and multiple: the phenotypic variance explained by each QTL and total variance explained by the nearest markers with the multiple regression model, respectively.

C955. In the vicinity of R1, Ray et al. (1996) detected a QTL for the tiller number (TN), Li et al. (1999) a QTL for the tiller angle, Sasahara et al. (1999) a QTL for the vascular bundle number (VBN) and Yagi et al. (2001) a QTL for the spikelet number per panicle. In the vicinity of R6, Ray et al. (1996) identified a QTL for the root number, Lu et al. (1996) a QTL for the grain number per panicle, Sasahara et al. (1999) a QTL for VBN, Yan et al. (1998) a QTL for plant height and Yamamoto et al. (2001) a QTL for CL and PnL. The large number of different QTLs identified indicates that the vicinities of R1 and R6 are important chromosomal regions for rice morphogenesis.

R2, R3, R10 and R11 had an effect (QTL) on FLL only, whereas R8 and R12 had an effect on FLW only. The QTLs with a single effect indicate that it is possible to change FLL and FLW separately and to modify the shape of the flag leaf. In R11, QTLs were detected for all the crop seasons, suggesting the stable expression of QTL across different environments. Kobayashi et al. (2003) identified QTLs for PnN, CL and PnL between C477-XNpb257, which was adjacent to R11. Ray et al. (1996), Yamamoto et al. (2001) and Xiao et al. (1996) also identified QTLs for TN, the 3rd internode length and PnL, respectively. These current and past results suggest that the vicinity of the centromere on chromosome 11 harbors many agronomically important genes, as in the case of R1 and R6. On the other hand, QTLs were detected 
Table 2. (continued)

\begin{tabular}{|c|c|c|c|c|c|c|c|c|c|c|c|}
\hline \multirow{2}{*}{ Trait } & \multirow{2}{*}{ Location } & \multirow{2}{*}{ Season } & \multirow{2}{*}{ Chr. } & \multirow{2}{*}{$\begin{array}{l}\text { Position } \\
\text { (cM) }\end{array}$} & \multirow{2}{*}{\multicolumn{2}{|c|}{ Marker interval }} & \multirow{2}{*}{$P$} & \multirow{2}{*}{ LOD } & \multirow{2}{*}{$\begin{array}{c}\alpha \\
\left(\mathrm{cm}^{\circ}{ }^{\circ}\right)\end{array}$} & \multicolumn{2}{|c|}{$\mathrm{R}^{2}(\%)$} \\
\hline & & & & & & & & & & Single & Multiple \\
\hline \multirow{24}{*}{ FLW } & \multirow{5}{*}{ JE } & \multirow{5}{*}{1997} & 1 & 24 & N079A & $R 210 B$ & 0.002 & 2.3 & 0.04 & 5.5 & \multirow{5}{*}{44.5} \\
\hline & & & 1 & 45 & C955 & $\overline{R 210 A}$ & $<0.001$ & 3.5 & 0.06 & 8.1 & \\
\hline & & & 4 & 150 & $\overline{X N p b 264}$ & XNpb197 & $<0.001$ & 11.6 & -0.09 & 24.5 & \\
\hline & & & 6 & 79 & $\overline{X N p b 12}$ & $X N p b 135$ & $<0.001$ & 3.6 & 0.06 & 8.4 & \\
\hline & & & 8 & 62 & $G 2132 B$ & $\overline{G 1073}$ & $<0.001$ & 2.7 & -0.05 & 6.3 & \\
\hline & \multirow{5}{*}{\multicolumn{2}{|c|}{$00 \mathrm{WS}$}} & 1 & 47 & C955 & $R 210 A$ & $<0.001$ & 4.6 & 0.06 & 10.7 & \multirow{5}{*}{47.6} \\
\hline & & & 4 & 150 & $\overline{X N p b 264}$ & XNpb197 & $<0.001$ & 12.4 & -0.08 & 26.0 & \\
\hline & & & 6 & 81 & $\overline{X N p b 12}$ & $X N p b 135$ & $<0.001$ & 2.8 & 0.04 & 6.5 & \\
\hline & & & 8 & 60 & $G 2132 B$ & $\overline{G 1073}$ & $<0.001$ & 2.9 & -0.04 & 6.9 & \\
\hline & & & 12 & 98 & $G 1106$ & $\underline{C 901}$ & $<0.001$ & 3.2 & -0.05 & 7.6 & \\
\hline & \multirow{14}{*}{ LB } & \multirow{7}{*}{ 01DS } & 1 & 25 & N079A & $R 210 B$ & $<0.001$ & 3.1 & 0.05 & 7.2 & \multirow{7}{*}{45.8} \\
\hline & & & 1 & 48 & C955 & $\overline{R 210 A}$ & $<0.001$ & 5.4 & 0.08 & 12.3 & \\
\hline & & & 4 & 150 & $X N p b 264$ & $X N p b 197$ & $<0.001$ & 9.1 & -0.08 & 19.7 & \\
\hline & & & 6 & 78 & $X N p b 12$ & $X N p b 135$ & 0.006 & 2.3 & 0.05 & 5.3 & \\
\hline & & & 8 & 61 & $G 2132 B$ & $\overline{G 1073}$ & $<0.001$ & 2.8 & -0.05 & 6.5 & \\
\hline & & & 10 & 50 & C16 & $X N p b 127$ & $<0.001$ & 3.0 & 0.05 & 7.0 & \\
\hline & & & 12 & 98 & $G 1106$ & $C 901$ & $<0.001$ & 3.0 & -0.05 & 6.9 & \\
\hline & & \multirow{7}{*}{$01 \mathrm{WS}$} & 1 & 25 & N079A & $R 210 B$ & $<0.001$ & 2.6 & 0.04 & 6.5 & \multirow{7}{*}{44.5} \\
\hline & & & 1 & 34 & $X N p b 359$ & $X N p b 90$ & 0.003 & 2.5 & 0.05 & 6.2 & \\
\hline & & & 1 & 48 & C955 & $R 210 A$ & 0.004 & 2.7 & 0.05 & 6.7 & \\
\hline & & & 3 & 77 & $\overline{C 136}$ & $X N p b 249$ & 0.004 & 2.8 & 0.05 & 7.0 & \\
\hline & & & 4 & 150 & $\overline{X N p b 264}$ & XNpb197 & $<0.001$ & 9.9 & -0.08 & 22.8 & \\
\hline & & & 6 & 77 & $\overline{X N p b 12}$ & $X N p b 135$ & $<0.001$ & 3.0 & 0.05 & 7.5 & \\
\hline & & & 12 & 98 & G1106 & $\underline{C 901}$ & 0.002 & 2.6 & -0.05 & 6.4 & \\
\hline \multirow{6}{*}{ FLA } & \multirow{2}{*}{$\mathrm{JE}$} & \multirow{2}{*}{1997} & 1 & 150 & \multicolumn{2}{|c|}{$C 112$} & 0.005 & 2.2 & -1.54 & 5.1 & \multirow{2}{*}{20.0} \\
\hline & & & 8 & 86 & XNpb187 & XNpb56 & $<0.001$ & 4.1 & -2.97 & 9.5 & \\
\hline & \multirow{4}{*}{ LB } & & 1 & 125 & $C 86$ & $X N p b 113$ & 0.003 & 2.6 & -2.42 & 6.5 & \\
\hline & & & 2 & 1 & XNpb349 & $N 366 U$ & 0.002 & 2.1 & 2.02 & 5.4 & \\
\hline & & 00DS & 4 & 132 & $X N p b 161$ & $X N p b 331$ & 0.003 & 2.4 & -2.43 & 6.0 & 11.6 \\
\hline & & & 7 & 2 & $X N p b 50$ & N1165R & $<0.001$ & 2.6 & -2.34 & 6.6 & \\
\hline
\end{tabular}

for R3, R10 and R12 in only Los Baños. This result demonstrates that the QTLs expressed by a tropical environment are involved in flag leaf development.

To conclude, flag leaf development was influenced by nine genomic regions categorized into three groups. In Group I, three regions (on chromosomes 1, 4 and 6) increased both FLL and FLW. The effects of these three regions, especially the region of chromosome 4, were very powerful and stable across the environments. In Group II, four regions (chromosomes 2, 3, 10 and 11) affected FLL, whereas in Group III, two regions (chromosomes 8 and 12) affected FLW. Among these six regions, three on chromosomes 3, 10 and 12 were expressed by a tropical environment. In this study, no region with a clear effect on FLA was identified, although this trait was segregated largely in the RILs. This implied that the erectness of the flag leaf was determined by the environments, rather than by genetic factors. These new findings suggest that (1) nearly a dozen genes, which have different functions, are involved in the genetic mechanisms of flag leaf development, (2) a few of them control the flag leaf size (FLL and FLW) and (3) some genes interact with environments and result in the genetic mecha- nisms differentiated among locations. The current results will be integrated with those for other yield-related traits to understand on the QTLs controlling rice yield. And, the QTLs for the flag leaf traits will be further studied by subsequent experiments using the substitution lines with the M23 or AK background.

\section{Acknowledgments}

This study was partly conducted under the IRRI-Japan Collaborative Research Project. We thank Messrs T. Aota, K. Yazaki and I. Nozaki, HNAES and Mr. L.A. Ebron, IRRI, for their skilled management of the paddy fields.

\section{Literature Cited}

Causse,M.A., T.M.Fulton, Y.G.Cho, S.N.Ahn, J.Chunwongse, K.Wu, J.Xiao, Z.Yu, P.C.Ronald, S.E.Harrington, G.Second, S.R. McCouch and S.D.Tanksley (1994) Saturated molecular map of the rice genome based on an interspecific backcross population. Genetics 138: 1251-1274.

Fukuta,Y., K. Tamura, H.Sasahara and T.Fukuyama (1999) Genetic 
and breeding analysis using molecular marker 18: Variations of gene frequency and the RFLP map of the hybrid population derived from the cross between the rice variety, Milyang 23 and Akihikari. Breed. Res. 1 (Suppl.2): 176.

Gladun,I.V. and E.A.Karpov (1993) Distribution of assimilates from the flag leaf of rice during the reproductive period of development. Russ. J. Plant. Physiol. 40: 215-219.

Kobayashi,S., Y.Fukuta, T.Sato, M.Osaki and G.S.Khush (2003) Molecular marker dissection of rice (Oryza sativa L.) plant architecture under temperate and tropical climates. Theor. Appl. Genet. (in press).

Li,Z., S.R.M.Pinson, J.W.Stansel and W.D.Park (1995) Identification of quantitative trait loci (QTLs) for heading date and plant height in cultivated rice (Oryza sativa L.). Theor. Appl. Genet. 91: 374-381.

Li,Z., S.R.M.Pinson, J.W.Stansel and A.H.Paterson (1998) Genetic dissection of the source-sink relationship affecting fecundity and yield in rice (Oryza sativa L.). Mol. Breed. 4: 419-426.

Li,Z., A.H.Paterson, S.R.M. Pinson and J.W. Stansel (1999) RFLP facilitated analysis of tiller and leaf angle in rice (Oryza sativa L.). Euphytica 109: 79-84.

Lin, H.X., H.R. Qian, J.Y.Zhuang, J.Lu, S.K.Min, Z.M.Xiong, N.Huang and K.L.Zheng (1996) RFLP mapping of QTLs for yield and related characters in rice (Oryza sativa L.). Theor. Appl. Genet. 92: 920-927.

Lu,C., L.Shen, Z.Tan, Y.Xu, P.He, Y.Chen and L.Zhu (1996) Comparative mapping of QTLs for agronomic traits of rice across environments using a doubled haploid population. Theor. Appl. Genet. 93: 1211-1217.

Nelson,J.C. (1997) QGENE: software for marker-based genomic analysis and breeding. Mol. Breed. 3: 239-245.

Ray,J.D., L.Yu, S.R.McCouch, M.C.Champoux, G.Wang and H.T. Nguyen (1996) Mapping quantitative trait loci associated with root penetration ability in rice (Oryza sativa L.). Theor. Appl. Genet. 92: 627-636.

Sasahara,H., Y.Fukuta and T.Fukuyama (1999) Mapping of QTLs for vascular bundle system and spike morphology in rice, Oryza sativa L. Breed. Sci. 49: 75-81.

Xiao,J., J.Li, L.Yan and S.D.Tanksley (1996) Identification of QTLs affecting traits of agronomic importance in a recombinant inbred population derived from a subspecific rice cross. Theor. Appl. Genet. 92: 230-244.

Yagi, T., K.Nagata, Y.Fukuta, K.Tamura, I. Ashikawa and T.Terao (2001) QTL mapping of spikelet number in rice (Oryza sativa L.). Breed. Sci. 51: 53-56.

Yamagata,H. (1997) Leaf. In "Science of the Rice Plant vol. III Genetics" Matsuo,T., Y.Futsuhara, F.Kikuchi and H.Yamaguchi (eds.), Food and Agriculture Policy Research Center, Tokyo. p. $277-285$.

Yamamoto,T., H.Lin, T.Sasaki and M.Yano (2000) Identification of heading date quantitative trait locus $H d 6$ and characterization of its epistatic interaction with $H d 2$ in rice using advanced backcross progeny. Genetics 154: 885-891.

Yamamoto,T., F.T.Shiobara, Y.Ukai, T.Sasaki and M.Yano (2001) Mapping quantitative trait loci for days-to-heading, and culm, panicle and internode lengths in $\mathrm{BC}_{1} \mathrm{~F}_{3}$ population using an elite rice variety, Koshihikari, as the recurrent parent. Breed. Sci. 51: 63-71.

Yan,J., J.Zhu, C.He, M.Benmoussa and P.Wu (1998) Molecular dissection of developmental behavior of plant height in rice (Oryza sativa L.). Genetics 150: 1257-1265.

Yan,J., J.Zhu, C.He, M.Benmoussa and P.Wu (1999) Molecular marker-assisted dissection of genotype $\times$ environment interaction for plant type traits in rice (Oryza sativa L.). Crop Sci. 39: 538-544.

Yano,M. and T.Sasaki (1997) Genetic and molecular dissection of quantitative traits in rice. Plant Mol. Biol. 35: 145-153.

Yokoo,M., S.Saito, T.Higashi and S.Matsumoto (1994) Use of a Korean rice cultivar Milyang 23 for improving Japanese rice. Breed. Sci. 44: 219-222.

Yonezawa,K. (1997) Yield components. In "Science of the Rice Plant vol. III Genetics" Matsuo, T., Y.Futsuhara, F. Kikuchi and H.Yamaguchi (eds.), Food and Agriculture Policy Research Center, Tokyo. p. 400-412. 